NUC-MINN-93/7-T

UMN-TH-1103/92

SUNY-NTG-92-21

MARCH, 1993

\title{
Neutron Stars and Nuclei in the Modified Relativistic Hartree Approximation
}

\author{
M. Prakash ${ }^{a, c}$, P. J. Ellis ${ }^{b, d}$, E. K. Heide ${ }^{b}$ and S. Rudaz ${ }^{b}$ \\ ${ }^{a}$ Theoretical Physics Institute, University of Minnesota, Minneapolis, MN 55455 \\ ${ }^{b}$ School of Physics and Astronomy, University of Minnesota, Minneapolis, MN 55455 \\ ${ }^{c}$ Physics Dept., State University of New York at Stony Brook, Stony Brook, NY 11794 \\ ${ }^{d}$ Institute for Nuclear Theory, University of Washington, Seattle, WA 98195
}

\begin{abstract}
We have examined the properties of neutron-rich matter and finite nuclei in the modified relativistic Hartree approximation for several values of the renormalization scale, $\mu$, around the standard choice of $\mu$ equal to the nucleon mass $M$. Observed neutron star masses do not effectively constrain the value of $\mu$. However for finite nuclei the value $\mu / M=0.79$, suggested by nuclear matter data, provides a good account of the bulk properties with a sigma mass of about $600 \mathrm{MeV}$. This value of $\mu / M$ renders the effective three and four body scalar self-couplings to be zero at $60 \%$ of equilibrium nuclear matter density, rather than in the vacuum. We have also found that the matter part of the exchange diagram has little impact on the bulk properties of neutron stars.
\end{abstract}




\section{Introduction}

The archetypal Walecka model is a point-particle, relativistic field theory in which nucleons interact via the exchange of $\sigma$-and $\omega$-mesons. If systems with non-zero isospin are studied the exchange of $\rho$-mesons also gives substantial contributions. This type of model has been widely employed to study matter both at normal and at high density e.g. [1]. The Lagrangian takes the form

$$
\begin{gathered}
\mathcal{L}=\bar{N}\left(i \gamma^{\mu} \partial_{\mu}-g_{\omega} \gamma^{\mu} \omega_{\mu}-\frac{g_{\rho}}{2} \gamma^{\mu} \mathbf{b}_{\mu} \cdot \boldsymbol{\tau}+\frac{f_{\rho}}{4 M} \sigma^{\mu \nu} \partial_{\nu} \mathbf{b}_{\mu} \cdot \boldsymbol{\tau}-M+g_{\sigma} \sigma\right) N \\
-\frac{1}{4} F_{\mu \nu} F^{\mu \nu}+\frac{1}{2} m_{\omega}^{2} \omega_{\mu} \omega^{\mu}-\frac{1}{4} \mathbf{B}_{\mu \nu} \cdot \mathbf{B}^{\mu \nu}+\frac{1}{2} m_{\rho}^{2} \mathbf{b}_{\mu} \cdot \mathbf{b}^{\mu} \\
+\frac{1}{2} \partial_{\mu} \sigma \partial^{\mu} \sigma-\frac{1}{2} m_{\sigma}^{2} \sigma^{2}
\end{gathered}
$$

where $F_{\mu \nu}=\partial_{\mu} \omega_{\nu}-\partial_{\nu} \omega_{\mu}, \mathbf{B}_{\mu \nu}=\partial_{\mu} \mathbf{b}_{\nu}-\partial_{\nu} \mathbf{b}_{\mu}$ and $\sigma^{\mu \nu}=\frac{1}{2} i\left[\gamma^{\mu}, \gamma^{\nu}\right]$. We have allowed for both vector and tensor coupling of the $\rho$ field, $\mathbf{b}_{\mu}$, to the nucleon (the latter is only employed in evaluating the exchange or Fock diagram). We note that the presence of the $\rho$ meson renders the Lagrangian non-renormalizable, although a renormalizable model can be constructed [1]].

In the mean field approximation, where the fields are replaced by their classical expectation value, the energy density for an infinite system is

$$
\begin{aligned}
\mathcal{E}_{\mathrm{MFA}}= & \frac{C_{\omega}^{2} n^{2}}{2 M^{2}}+\frac{M^{2}}{2 C_{\sigma}^{2}}\left(M^{*}-M\right)^{2}+\frac{C_{\rho}^{2}}{8 M^{2}}\left(n_{N}-n_{P}\right)^{2} \\
& +\frac{1}{\pi^{2}} \int_{0}^{k_{F N}} d k k^{2} E^{*}+\frac{1}{\pi^{2}} \int_{0}^{k_{F P}} d k k^{2} E^{*} .
\end{aligned}
$$

Here the nucleon effective mass, $M^{*}=M-g_{\sigma} \sigma, E^{*}=\sqrt{k^{2}+M^{* 2}}$ and $C_{i}^{2}=\left(g_{i} M / m_{i}\right)^{2}$, where $i$ denotes the meson species. The subscripts $N$ and $P$ on the Fermi momentum $k_{F}$ and density $n$ distinguish the neutron and 
proton values. The total density $n=n_{N}+n_{P}$, thus the neutron excess parameter $\alpha=\frac{N-Z}{A}=\frac{n_{N}-n_{P}}{n}$. Normal nuclear matter corresponds to $\alpha=0$, but here we shall be interested in neutron star matter for which $\alpha \sim 1$. Also matter with $\alpha \sim \frac{1}{3}$ is of interest for stellar collapse leading to supernova explosions.

In the relativistic Hartree approximation (RHA), where the one loop diagrams are taken into account, the energy density is the sum of the mean field contribution and a one-loop vacuum correction term $\mathcal{E}_{\mathrm{RHA}}=\mathcal{E}_{\mathrm{MFA}}+\Delta \mathcal{E}$. The one-loop vacuum correction term, caused by a shift in the single particle energies, receives contributions from the $\sigma$-interaction only. It contains divergences and the necessary renormalization introduces a scale, $\mu$. Explicitly [2],

$$
\Delta \mathcal{E}\left(M^{*}\right)=-\frac{1}{4 \pi^{2}}\left(M^{* 4} \ln \frac{M^{*}}{\mu}+\sum_{n=0}^{4} f_{n} M^{4-n}\left(M-M^{*}\right)^{n}\right) .
$$

In order to specify the finite parameters $f_{n}$, we define $\lambda_{m}\left(M^{*}\right)=\frac{d^{m} \Delta \mathcal{E}\left(M^{*}\right)}{d M^{* m}}$ and require $\lambda_{0}(M)$ and $\lambda_{1}(M)$ to be zero so that the vacuum energy is zero and corresponds to a minimum. We can also set $\lambda_{2}\left(M^{*}\right)=0$ at $M^{*}=M$ because different choices simply correspond to modifying the parameter $C_{\sigma}^{2}$ which is fitted to equilibrium nuclear matter. The remaining parameters are specified by requiring that $\lambda_{3}\left(M^{*}\right)$ and $\lambda_{4}\left(M^{*}\right)$, the effective three and four body couplings, are zero at some scale and for simplicity we choose the same scale $M^{*}=\mu$ in both cases. This yields [2]

$$
\begin{aligned}
f_{0}=\ln (\mu / M), & f_{1}=1-4 \ln (\mu / M), \\
f_{2}=-\frac{7}{2}+6 \ln (\mu / M), & f_{3}=\frac{25}{3}-4 \mu / M, \quad f_{4}=-\frac{25}{12} .
\end{aligned}
$$


The vacuum correction term $\Delta \mathcal{E}$ can then be written in the form

$$
\begin{aligned}
\Delta \mathcal{E}=-\frac{M}{\pi^{2}}(1- & \left.\frac{\mu}{M}+\ln \frac{\mu}{M}\right)\left(M-M^{*}\right)^{3} \\
& +\frac{1}{4 \pi^{2}} \ln \frac{\mu}{M}\left(M-M^{*}\right)^{4}+\Delta \mathcal{E}_{\mathrm{CW}} .
\end{aligned}
$$

For the standard choice [1], $\mu=M$, the first and second terms on the right vanish and one is left with the Chin-Walecka [3] result

$$
\begin{gathered}
\Delta \mathcal{E}_{\mathrm{CW}}=-\frac{1}{4 \pi^{2}}\left[M^{* 4} \ln \frac{M^{*}}{M}+M^{3}\left(M-M^{*}\right)-\frac{7}{2} M^{2}\left(M-M^{*}\right)^{2}\right. \\
\left.+\frac{13}{3} M\left(M-M^{*}\right)^{3}-\frac{25}{12}\left(M-M^{*}\right)^{4}\right] .
\end{gathered}
$$

Note that eq. (6) is designed to have no $\sigma^{3}$ or $\sigma^{4}$ contribution, such terms arise for $\mu \neq M$ and are shown explicitly in eq. (5). We refer to this approach as the modified relativistic Hartree approximation (MRHA).

In principle the results of an exact treatment would be independant of the renormalization scale, however this will not be the case when a simple approximation such as the MRHA is employed. Therefore the renormalization scale should be chosen on physical grounds and we have previously found [2, 4] that in nuclear matter the choice $\mu \neq M$ gives a better account of the second derivative of the energy/particle at saturation (the compression modulus) and also the third derivative (the skewness or anharmonicity). Our purpose here is to test the MRHA further by applying it to finite nuclei and neutron stars. Nuclei are expected to provide a more stringent test, in particular they are much more sensitive to the value of the nucleon effective mass than infinite systems. The value of $M^{*}$ in the interior of a heavy nucleus should approximate that found for equilibrium nuclear matter, which varies significantly with the renormalization scale. We will examine the predic- 
tions of the MRHA for the spherically-symmetric, doubly-closed-shell nuclei ${ }^{16} \mathrm{O},{ }^{40} \mathrm{Ca}$ and ${ }^{208} \mathrm{~Pb}$. In the case of neutron stars we are dealing with dense asymmetric matter. Apart from the obvious question of whether the MRHA supports neutron stars of the observed mass, we ask whether such global properties provide a significant constraint on the nuclear equation of state around the saturation density. It is also of interest to ask whether the proton fractions are such that the direct Urca process, i.e. neutrino emission from a single nucleon, is possible. If so, this will provide the dominant cooling mechanism [5] since the standard modified Urca mechanism involves two nucleons.

Exchange terms are a fundamental feature of nuclear physics, but they are not included in the MRHA. An additional goal of this work is to study the contribution of the lowest-order, two-loop exchange diagram to nuclear and neutron-rich matter. The vacuum contributions are large in magnitude, however it has been found that they become small if form factors are included to reflect the composite nature of the particles [6]. We therefore consider just the matter, or Fermi sea, contributions which are much less affected by form factors. This is a tractable calculation which should allow us to get a qualitative indication of the importance of exchange effects in dense matter.

The organization of the paper is as follows. Section 2 deals with the MRHA; we start with a brief discussion of nuclear matter in subsec. 2.1, turn to asymmetric matter in subsec. 2.2, neutron stars in subsec. 2.3. and, finally, finite nuclei in subsec. 2.4. In sec. 3 we investigate the effect of the matter part of the lowest-order exchange diagram for infinite systems. Our conclusions are given in sec. 4 . 


\section{The Modified RHA}

\subsection{Nuclear Matter}

For a given value of $\mu / M$ the parameters $C_{\omega}^{2}$ and $C_{\sigma}^{2}$ are fitted to the equilibrium nuclear matter properties. We take a binding energy/particle of 16 $\mathrm{MeV}$. In the interior region of heavy nuclei the measured charge density indicates an equilibrium matter density of $n_{0}=0.16 \pm 0.01 \mathrm{fm}^{-3}$; we choose the central value of $0.16 \mathrm{fm}^{-3}$. The parameter $C_{\rho}^{2}$ is fitted to the coefficient of the symmetry energy, $a_{\text {symm }}=\left.\frac{1}{2 n} \frac{\partial^{2} \mathcal{E}}{\partial \alpha^{2}}\right|_{\alpha=0}$, which is taken to be $30 \mathrm{MeV}$. The resulting parameters are listed in table 1 for a range of values of $\mu / M$. The omega coupling constants, $C_{\omega}^{2}$, agree qualitatively with the experimental result [7] of $103 \pm 36$ and are within the cited errors for $\mu / M=0.73,1.00$ and 1.25. Table 1 also gives the calculated compression moduli, $K=k_{F}^{2} \frac{d^{2}}{d k_{F}^{2}}\left(\frac{E}{A}\right)$. However, Pearson [8] has made the point that $K$ is not uniquely determined by the limited amount of accurate breathing mode data available (see also Shlomo and Youngblood 9]). He determines a linear correlation between $K$ and the coefficient of the Coulomb term, $K_{\mathrm{coul}} Z^{2} / A^{\frac{4}{3}}$, in the leptodermous expansion. This coefficient can be conveniently written

$$
K_{\text {coul }}=-\frac{3}{5} \frac{e^{2}}{r_{0}}\left[2+\frac{S}{K}\right]
$$

where $r_{0}$ is the radius parameter and $S$ is the skewness or anharmonicity

parameter defined by $S=k_{F}^{3} \frac{d^{3}}{d k_{F}^{3}}\left(\frac{E}{A}\right)$. One then has a linear correlation between $S / K$ and $K$ as shown by the shaded band in fig. 1 (the box indicates the point obtained by Sharma [10], however $\chi^{2}$ changes little over the shaded area). The points give results [4] obtained in the MRHA for various values 
of the renormalization scale, $\mu$. The MRHA results lie within the error band for values of the renormalization scale $\approx 0.79$ and 1.25 . The other values of $\mu / M$ in table 1 lie off the shaded band and are included in our discussion for comparison purposes. The value 0.73 has the attractive feature that it is the self-consistent point where $\mu=M_{\text {sat }}^{*}$.

\section{$2.2 \quad$ Asymmetric Matter}

We show in fig. 2 the binding energy/particle for nuclear matter and for pure neutron matter as a function of density; the full curves are obtained in the present MRHA approximation. At a given density the difference in energy between the pairs of curves is roughly independent of $\mu$, varying by less than $10 \%$. As regards the stiffness of the equation of state, it is often assumed that this can be deduced qualitatively from the compression modulus. We see, however, that it is not always a reliable indicator. Thus at high density the equation of state for $\mu=0.73 M$ is noticably stiffer than for $\mu=1.25 M$, yet the compression modulus is smaller. A quantitative measure of the high density behavior of the equation of state is provided by the adiabaticity index, $\Gamma=(d \ln P / d \ln n)$. At densities $n>2.5 n_{0}$ the value of $\Gamma$ for both nuclear and neutron matter lies in the range $2-3$, with little dependence on the renormalization scale apart from the case $\mu=0.73 M$. Here the pressure increases more rapidly with density and $\Gamma$ is significantly larger than in the other cases.

As we add neutrons to nuclear matter, i.e., as $\alpha$ increases from 0 , the equilibrium binding energy is reduced and the corresponding density is reduced. 
We have verified that this can be accurately described by the expression 11

$$
n_{\mathrm{sat}}(\alpha)=n_{\mathrm{nm}}\left(1-C \alpha^{2}\right)
$$

The constant $C$ is listed in Table 2. Further one can define the compression modulus at the saturation density of the asymmetric system, i.e., the isobaric incompressibility $K_{\text {iso }}$, and to second order in $\alpha$ this can be written

$$
K_{\text {iso }}(\alpha)=K\left[1+(A-B) \alpha^{2}\right] \equiv K\left[1-\widetilde{A} \alpha^{2}\right]
$$

Here the coefficient $A$ gives the asymmetry dependence of $K_{\text {iso }}$ at nuclear matter density, while $B$ allows for the change of equilibrium density in the neutron-rich system. These parameters, along with the combination $\widetilde{A}$ are given in table 2. Again we remark that this quadratic form provides a very accurate approximation, excepting only values of $\alpha$ close to the point where the system becomes unbound. The values of $C$ and $\widetilde{A}$ are smallest for $\mu / M=1$ and show a factor of 3 variation with renormalization scale. Other calculations [11] show a rather wide variation in the predicted values of $C$. However $\widetilde{A}$ is close to 1.6 in the chiral $\sigma$-model and it usually lies in the range $1.3-2.2$ in non-relativistic calculations, although larger values are to be found. These are comparable to the values in table 2 .

\subsection{Stellar Matter}

We have calculated the properties of neutron stars using the hydrostatic equilibrium equations of Tolman, and Oppenheimer and Volkov [13]. For $n>0.08 \mathrm{fm}^{-3}$, the MRHA equation of state is used. For densities $0.001<$

$n<0.08 \mathrm{fm}^{-3}$, we employ the equation of state of Negele and Vautherin [14], 
while for very low densities $\left(n<0.001 \mathrm{fm}^{-3}\right)$, we use the Baym-PethickSutherland [15] equation of state. The calculation is carried out for charge neutral matter in beta equilibrium. The condition for beta equilibrium is

$$
\mu_{e}=\mu_{N}-\mu_{P}=-\frac{\partial E(n, x)}{\partial x}
$$

where $\mu_{i}, i=N, P$ and $e$, are the chemical potentials of the fermions, $x=$ $Z / A$ and $E(n, x)$ is the energy per baryon. Muons will be present when $\mu_{e} \geq$ $m_{\mu}=105.7 \mathrm{MeV}$, which is generally the case for $n \geq n_{0}$. In the presence of muons, the proton fractions are determined by imposing the charge neutrality condition $n_{e}+n_{\mu}=n_{P}$ and the energy conservation condition $\mu_{e}=\mu_{\mu}$. The lepton contributions to the energy density and pressure are given by Fermi gas expressions (the electromagnetic interactions of the leptons give negligible contributions).

The measured mass [16] of $4 \mathrm{U} 0900-40$, which is $(1.85 \pm 0.3) M_{\odot}$, may provide a limit for the maximum mass of a neutron star. The most accurate determination [17], for one component of the binary pulsar PSR $1913+16$, is $(1.44 \pm 0.01) M_{\odot}$. The maximum masses in table 3 are consistent with these limits, although since hyperons are expected to reduce the maximum mass [18, 19] the case with $\mu=1.5 M$ can probably be excluded and the value for $\mu=1.25 M$ appears dangerously low. Note that $M_{\max }$ is not a monotonic function of $K$. Thus observed neutron star masses cannot be used to effectively constrain the compression modulus alone. Such properties of nuclear matter are not uniquely determined by the gross features of neutron stars [20], which constrain the softness of the neutron-rich equation of state. In table 3 we also give the radius of the neutron star and the ratio of the cen- 
tral density to equilibrium nuclear matter density, the latter for a maximum mass star and a $1.44 M_{\odot}$ star. The qualitative trends follow the high density stiffness of the equation of state.

We turn now to estimate the maximum Keplerian frequency of rotation from the formula 21]

$$
\Omega_{K} \simeq 0.77\left(\frac{M_{\max }}{M_{\odot}}\right)^{\frac{1}{2}}\left(\frac{R_{\max }}{10 \mathrm{~km}}\right)^{-\frac{3}{2}} 10^{4} \mathrm{~s}^{-1}
$$

where $M_{\max }$ and $R_{\max }$ are the maximum mass and corresponding radius for the spherical non-rotating star. The empirical relation (11) reproduces the results of more exact calculations [22, 23]. It is interesting to observe that changes in the renormalization scale serve to increase the Keplerian rotation frequency and give values which are on the upper end of those found with relativistic equations of state [23].

Recently, it has been pointed out [5] that the direct Urca processes

$$
n \rightarrow p+e^{-}+\bar{\nu}_{e} \quad, \quad p+e^{-} \rightarrow n+\nu_{e}
$$

may constitute the principal avenues through which the late-time rapid cooling of neutron stars occurs. For these processes to occur in matter in which the only baryons are nucleons, momentum conservation requires that the magnitude of the electron concentration $x_{e}$ exceeds a value [5]

$$
\left|x_{e}\right|^{1 / 3} \geq\left|(1-x)^{1 / 3}-x^{1 / 3}\right|
$$

where $x=Z / A$ is the proton fraction. When leptons $\left(e^{-}\right.$and $\left.\mu^{-}\right)$are the only source of negatively charged particles, charge neutrality and eq. (13) stipulate that $x$ exceed a value in the range $0.11-0.15$. In the models 
considered above, the symmetry energy rises sufficiently rapidly with density so that the calculated proton fractions are large enough for the direct Urca cooling mechanism to be operative.

Since the calculated maximum neutron star masses exceed the observed values for all but very large values of $\mu / M$, we conclude that the gross properties of neutron stars do not effectively constrain the renormalization scale $\mu / M$.

\section{$2.4 \quad$ Finite Nuclei}

\subsubsection{Formalism}

For finite nuclei the Coulomb interaction is needed so that the Lagrangian (1) is supplemented by the photon Lagrangian

$$
\mathcal{L}_{p h}=-\frac{1}{4} f_{\mu \nu} f^{\mu \nu}-e \bar{N} \frac{1}{2}\left(1+\tau_{3}\right) \gamma^{\mu} A_{\mu} N
$$

Here $A_{\mu}$ is the Maxwell field and $f_{\mu \nu}$ is the electromagnetic field strength tensor. It is straightforward [1, 24] to obtain from $\mathcal{L}+\mathcal{L}_{p h}$ the equations of motion in the mean field approximation. The vacuum is taken into account by means of the local density approximation and the leading correction terms of the derivative expansion [25]. The latter give small, although not negligible corrections 26]. The derivative expansion appears to be rapidly convergent [27, 28] so that the lowest order corrections should suffice.

The corrections of the derivative expansion can be obtained by evaluating the one-loop polarization contribution to the scalar and vector meson propagators [29]. The question arises as to where these propagators should be 
renormalized, at $M^{*}=M$ or at $M^{*}=\mu$. If in the former case one chooses $\lambda_{2}(M)=0$ and in the latter $\lambda_{2}(\mu)=0$, where $\lambda_{2}$ is defined in sec. 1 , then changing the renormalization point for the scalar meson is simply equivalent to varying $m_{\sigma}$, which is a free parameter anyway. For the $\omega$-meson it is reasonable to require that, in vacuum, the pole of the propagator lies at the known physical mass. Then changes in the renormalization point will not affect the predictions, but rather the interpretation of the parameters. Therefore it is sufficient to take the derivative contributions in standard form [25] So that the complete scalar density is

$$
\begin{aligned}
\rho_{s}(r) & =\sum_{\alpha}^{\text {occ }} \frac{\left(2 j_{\alpha}+1\right)}{4 \pi r^{2}}\left[G_{\alpha}(r)^{2}-F_{\alpha}(r)^{2}\right]+\frac{d \Delta \mathcal{E}}{d M^{*}} \\
& -\frac{1}{4 \pi^{2}}\left[2 g_{\sigma} \ln \frac{M^{*}}{M}\left(\frac{d^{2} \sigma}{d r^{2}}+\frac{2}{r} \frac{d \sigma}{d r}\right)-\frac{g_{\sigma}^{2}}{M^{*}}\left(\frac{d \sigma}{d r}\right)^{2}-\frac{2 g_{\omega}^{2}}{3 M^{*}}\left(\frac{d \omega_{0}}{d r}\right)^{2}\right]
\end{aligned}
$$

where $G$ and $F$ are the components of the Dirac spinors for the occupied states and $M^{*}$ is the spatially dependant effective mass, $M^{*}(r)=M-g_{\sigma} \sigma(r)$. Only the timelike component of $\omega_{\mu}$ is non-zero for the spherically symmetric nuclei we consider. The full vector density is

$$
\begin{aligned}
\rho_{B}(r)= & \sum_{\alpha}^{\text {occ }} \frac{\left(2 j_{\alpha}+1\right)}{4 \pi r^{2}}\left[G_{\alpha}(r)^{2}+F_{\alpha}(r)^{2}\right] \\
& -\frac{1}{3 \pi^{2}}\left[g_{\omega} \ln \frac{M^{*}}{M}\left(\frac{d^{2} \omega_{0}}{d r^{2}}+\frac{2}{r} \frac{d \omega_{0}}{d r}\right)-\frac{g_{\sigma} g_{\omega}}{M^{*}}\left(\frac{d \sigma}{d r} \frac{d \omega_{0}}{d r}\right)\right],
\end{aligned}
$$

and the contribution to the total energy from the vacuum is given by

$$
\Delta E=4 \pi \int_{0}^{\infty} r^{2} d r\left[\Delta \mathcal{E}\left(M^{*}\right)-\frac{g_{\sigma}^{2}}{4 \pi^{2}} \ln \frac{M^{*}}{M}\left(\frac{d \sigma}{d r}\right)^{2}+\frac{g_{\omega}^{2}}{6 \pi^{2}} \ln \frac{M^{*}}{M}\left(\frac{d \omega_{0}}{d r}\right)^{2}\right]
$$

where $M^{*}=M^{*}(r)$. 


\subsubsection{Results}

We now consider the predictions of the MRHA for finite nuclei 1 , specifically the doubly closed-shell nuclei ${ }^{16} \mathrm{O},{ }^{40} \mathrm{Ca}$ and ${ }^{208} \mathrm{~Pb}$. In addition to the parameters of table 1, the meson masses are required. We use the physical masses for the $\rho$ - and $\omega$-mesons; the value of $m_{\sigma}$, which is not a priori known, is discussed below. A pure vector $\rho N$ coupling is employed in these calculations. As we have remarked, the vacuum is treated by means of the local density approximation supplemented by the leading terms of the derivative expansion. The effect of the latter are fairly small, giving a decrease in the binding energy, an increase in the radius for a fixed $\sigma$ mass and very little change in the single particle energies. If $m_{\sigma}$ is adjusted so that similar radii are obtained with and without the derivative terms, the net result is a small increase in the binding energy when the derivative terms are included [26]. We shall present results which include the contributions of the derivative terms.

In table 4 we summarize the bulk properties of the nuclei as a function of $\mu / M$ for a fixed value of $m_{\sigma}=550 \mathrm{MeV}$. The theoretical values of the binding energy/particle include a correction for the c.m. kinetic energy [31]. The charge densities, and therefore the radii quoted, are corrected for the finite size of the proton [24] and for c.m. effects [32]. In table 4 we also give the separation between the neutron $2 f$ levels in ${ }^{208} \mathrm{~Pb}$ so as to indicate the order of magnitude of the spin-orbit splittings. In studying table 4 it must

\footnotetext{
${ }^{1}$ This is also under investigation by Blunden [30] with similar results where comparison can be made.
} 
be borne in mind that while $C_{\sigma}^{2}$ is fixed from nuclear matter, the value of $m_{\sigma}$ can be regarded as a free parameter. Decreasing $m_{\sigma}$ has the effect of increasing $r_{c h}$ and decreasing $\frac{B E}{A}$ and vice versa [24]. Thus for $\mu / M=0.73$ the charge radius of $\mathrm{Pb}$ is too large, while those of $\mathrm{O}$ and $\mathrm{Ca}$ are too small so that we shall not be able to obtain a reasonable account of these nuclei by varying $m_{\sigma}$. For $\mu / M=1.5$, the deviations of the radius from experiment are quite disparate for $\mathrm{Ca}$ and $\mathrm{Pb}$ and this cannot be resolved by adjusting $m_{\sigma}$. Further in this case the spin-orbit splittings are much too small- the value in table 4 is less than a third of the experimental figure. This is due to the large value of the effective mass at saturation in nuclear matter (0.86). As is well known [1] smaller effective masses $\sim 0.6$ yield better values for the spin-orbit splittings and this is evident from comparison of tables 1 and 4. The value $\mu / M=1.25$ also yields quite small spin-orbit splittings due to a large value of $M_{\text {sat }}^{*}$, although, for comparison purposes, we will consider it along with the values 0.79 and 1.0 in subsequent calculations.

The results obtained by varying $m_{\sigma}$ so as to approximately fit the radius of $\mathrm{Ca}$ and, if possible, also $\mathrm{Pb}$ are given in table 5. The binding energies for $\mu / M=0.79$ are in strikingly good agreement with the data for a reasonable value of $m_{\sigma}=600 \mathrm{MeV}$. With $\mu / M=1.25$ the binding energies are low and the required $\sigma$ mass is unreasonably small. The value $\mu / M=1.0$ also yields rather low binding energies and further one cannot obtain a good account of the charge radii of $\mathrm{Ca}$ and $\mathrm{Pb}$ simultaneously. We should emphasize at this juncture that our results are obtained by fitting to a nuclear matter saturation density, $n_{0}=0.16 \mathrm{fm}^{-3}$. A number of other authors, e.g. [1, 24, 26, 33], have used the value $0.15 \mathrm{fm}^{-3}$ which represents a reduction in $k_{F}$ of $2 \%$. This 
has the effect of increasing the nuclear radius by approximately $2 \%$ so that a larger $m_{\sigma}$ can be used and this increases the binding energies. For example, Wasson [26] takes $550 \mathrm{MeV}(\mu / M=1.0)$ which gives binding energies 1-2 $\mathrm{MeV}$ short of the experimental value and in fact for $\mu / M=0.79$ the nuclei are overbound with this saturation density. In both cases close agreement with the observed radii for all three nuclei cannot be obtained.

Adopting the values of $m_{\sigma}$ in table 5 which are appropriate to $n_{0}=0.16$ $\mathrm{fm}^{-3}$, we obtain the single particle energies of ${ }^{208} \mathrm{~Pb}$ which are shown in figs. 3 and 4 for neutrons and protons respectively. We indicate both occupied and unoccupied experimental states and their theoretical counterparts, if bound, for levels near the Fermi energy. The shell closure and rough level ordering are reproduced quite well, however the occupied neutron (proton) levels are too strongly (weakly) bound which could be improved by increasing the $\rho$ coupling [24]. For example an increase in $g_{\rho}^{2}$ of $40 \%$, corresponding to a symmetry energy of $35 \mathrm{MeV}$, would shift the occupied single particle levels in the right direction by about $1 \mathrm{MeV}$; the unoccupied levels are shifted by a somewhat smaller amount.

The spin orbit splittings that we find are too small; on average they are 80,60 and $30 \%$ of the experimental values for $\mu / M=0.79,1.0$ and 1.25 , respectively, and the percentage becomes somewhat smaller for the lighter nuclei. For the standard renormalization, Fox [33] has made the same point and noted that the mean field results are close to, or a little larger than, the data (see also, for example, the fits in ref. [34]). Finally we show in figs. 5-7 the comparison of the predicted charge distributions with the data (experimental errors are not indicated since they are negligible except near 
the center of the nuclei). In $\mathrm{Pb}$ for $\mu / M=1.0$ our central density is too high, whereas Fox [33] achieves better agreement, presumably due to his choice of $n_{0}=0.15 \mathrm{fm}^{-3}$. We do however find a region of positive slope, although it not as pronounced as the data; mean field calculations do better in this respect. Our results for $\mu / M=0.79$ and 1.25 are significantly better, both for radial distances of $2-5 \mathrm{fm}$ and in the important tail region. For $\mathrm{Ca}, \mu / M=1.0$ clearly gives the best agreement with the data, although the other cases are not unreasonable. In $\mathrm{O}$ the different theoretical results are of comparable quality.

Viewing the results for finite nuclei overall, the renormalization scale $\mu / M=0.79$ gives the best representation of the data. The additional terms which are introduced into the vacuum correction $\Delta \mathcal{E}$ were given in eq. (5). This value of $\mu / M$ renders the self-consistently determined effective three and four body scalar self-couplings to be zero at a nuclear density of $n=0.092$ $\mathrm{fm}^{-3}$, which is roughly halfway between the equilibrium point and the vacuum. With $\mu / M=0.79$ the coefficient of the induced $\sigma^{4}$ term is negative and this has long been known to be necessary in mean field treatments which fit parameters of $\sigma^{3}$ and $\sigma^{4}$ terms to nuclei [34, 35, 36]. 


\section{Exchange Contributions for Infinite Systems}

\subsection{Formalism}

We turn now to assess the importance of exchange contributions from the two-loop diagram shown in fig. 8; here a solid line represents a nucleon with propagator $S$ and a dashed line represents a meson with propagator $D$. Along with the $\sigma$ and $\omega$ mesons we shall include the $\rho$ since this is clearly necessary for neutron-rich matter. The exchange diagram gives a contribution to the energy density

$$
\mathcal{E}=\frac{1}{2} \int \frac{d^{4} p_{1}}{(2 \pi)^{4}} \frac{d^{4} p_{2}}{(2 \pi)^{4}} \operatorname{Tr}\left[S\left(p_{1}\right) S\left(p_{2}\right)\right] D(k),
$$

where $k=p_{1}-p_{2}$ and Lorentz indices have been suppressed. We use the mean field form of the propagators, treating the two-loop contribution as a perturbation. The matter part of this contribution to the energy density involves the basic integrals

$$
\begin{aligned}
& I\left(w, \xi_{1}, \xi_{2}\right)=\int_{1}^{\xi_{1}} d u\left(1-\frac{1}{u^{2}}\right) J\left(w, u, \xi_{2}\right), \\
& J\left(w, u, \xi_{2}\right)=\frac{1}{4} \int_{1}^{\xi_{2}} d v\left(1-\frac{1}{v^{2}}\right) \ln \frac{(u v-1)^{2}+u v w}{(u-v)^{2}+u v w} .
\end{aligned}
$$

For completeness, we first give the expressions [3] for $\sigma$ - and $\omega$-exchange. The energy density is the sum of neutron and proton contributions

$$
\begin{aligned}
& \mathcal{E}_{\sigma}=e_{\sigma}\left(k_{F N}\right)+e_{\sigma}\left(k_{F P}\right), \\
& \mathcal{E}_{\omega}=e_{\omega}\left(k_{F N}\right)+e_{\omega}\left(k_{F P}\right) .
\end{aligned}
$$


Here

$$
\begin{aligned}
& e_{\sigma}\left(k_{F}\right)=\frac{g_{\sigma}^{2}}{64 \pi^{4}}\left[\left(k_{F} E_{F}^{*}-M^{* 2} \ln \xi\right)^{2}+M^{* 4}\left(4-w_{\sigma}\right) I\left(w_{\sigma}, \xi, \xi\right)\right], \\
& e_{\omega}\left(k_{F}\right)=\frac{g_{\omega}^{2}}{32 \pi^{4}}\left[\left(k_{F} E_{F}^{*}-M^{* 2} \ln \xi\right)^{2}-M^{* 4}\left(2+w_{\omega}\right) I\left(w_{\omega}, \xi, \xi\right)\right],
\end{aligned}
$$

where

$$
\begin{aligned}
E_{F}^{* 2} & =k_{F}^{2}+M^{* 2}, \quad \xi=\left(k_{F}+E_{F}^{*}\right) / M^{*}, \\
w_{\sigma} & =\left(m_{\sigma} / M^{*}\right)^{2} \quad \text { and } \quad w_{\omega}=\left(m_{\omega} / M^{*}\right)^{2} .
\end{aligned}
$$

The energy density for $\rho$ exchange can be written

$$
\mathcal{E}_{\rho}=e_{\rho}\left(k_{F N}, k_{F N}\right)+e_{\rho}\left(k_{F P}, k_{F P}\right)+4 e_{\rho}\left(k_{F N}, k_{F P}\right) .
$$

For the general case with both vector and tensor coupling each $e_{\rho}$ is the sum of vector-vector, tensor-tensor and vector-tensor contributions

$$
e_{\rho}\left(k_{F 1}, k_{F 2}\right)=e_{\rho}^{\mathrm{vv}}\left(k_{F 1}, k_{F 2}\right)+e_{\rho}^{\mathrm{tt}}\left(k_{F 1}, k_{F 2}\right)+e_{\rho}^{\mathrm{vt}}\left(k_{F 1}, k_{F 2}\right) .
$$

These contributions can be written in the form

$$
\begin{gathered}
e_{\rho}^{\mathrm{vv}}\left(k_{F 1}, k_{F 2}\right)=\left(\frac{g_{\rho}}{2}\right)^{2} \frac{1}{32 \pi^{4}}\left[\left(k_{F 1} E_{F 1}^{*}-M^{* 2} \ln \xi_{1}\right)\left(k_{F 2} E_{F 2}^{*}-M^{* 2} \ln \xi_{2}\right)\right. \\
\left.-M^{* 4}\left(2+w_{\rho}\right) I\left(w_{\rho}, \xi_{1}, \xi_{2}\right)\right], \\
e_{\rho}^{\mathrm{tt}}\left(k_{F 1}, k_{F 2}\right)=\left(\frac{f_{\rho}}{4 M}\right)^{2} \frac{M^{* 2}}{64 \pi^{4}}\left[\left(10+w_{\rho}\right)\left(k_{F 1} E_{F 1}^{*}-M^{* 2} \ln \xi_{1}\right)\right. \\
\left.\times\left(k_{F 2} E_{F 2}^{*}-M^{* 2} \ln \xi_{2}\right)-\frac{8 k_{F 1}^{3} k_{F 2}^{3}}{9 M^{* 2}}-M^{* 4} w_{\rho}\left(8+w_{\rho}\right) I\left(w_{\rho}, \xi_{1}, \xi_{2}\right)\right], \\
e_{\rho}^{\mathrm{vt}}\left(k_{F 1}, k_{F 2}\right)=\left(\frac{g_{\rho} f \rho}{8 M}\right) \frac{3 M^{*}}{16 \pi^{4}}\left[\left(k_{F 1} E_{F 1}^{*}-M^{* 2} \ln \xi_{1}\right)\left(k_{F 2} E_{F 2}^{*}-M^{* 2} \ln \xi_{2}\right)\right. \\
\left.-M^{* 4} w_{\rho} I\left(w_{\rho}, \xi_{1}, \xi_{2}\right)\right],
\end{gathered}
$$


where $w_{\rho}=\left(m_{\rho} / M^{*}\right)^{2}$ and the subscripts 1 and 2 on $E_{F}$ and $\xi$ refer to the appropriate Fermi momenta. If the no-retardation approximation is made, i.e., energy differences $\left(E_{1}^{*}-E_{2}^{*}\right)$ are set to zero, this can be put in the form given by Bouyssy et al. [12]. Note that they remove the (three-dimensional) delta function contribution from the tensor-tensor piece, arguing that it should be suppressed by short-range correlations.

Thus in this approximation the total energy density $\mathcal{E}_{\text {total }}=\mathcal{E}_{\mathrm{RHA}}+\mathcal{E}_{\text {exch }}$, where $\mathcal{E}_{\text {exch }}=\mathcal{E}_{\sigma}+\mathcal{E}_{\omega}+\mathcal{E}_{\rho}$. For a given density the value of $M^{*}$ is obtained from the usual minimization condition $\partial \mathcal{E}_{\text {total }} / \partial M^{*}=0$ and here both the RHA and exchange contributions are taken into account. A word of caution is in order regarding the high density behavior, $k_{F} \rightarrow \infty$. Since in this limit $\mathcal{E}_{\mathrm{MFA}} \propto n^{2}$, while $\mathcal{E}_{\sigma}$ and $\mathcal{E}_{\omega} \propto n^{\frac{4}{3}}$ [3] these exchange contributions are negligible. However the $\rho$ tensor coupling involves a derivative which brings in an extra power of the momentum. This causes the tensor-tensor contribution to dominate the asymptotic behavior so that $\mathcal{E}_{\rho} \propto-n^{2}$ and the total energy density $\mathcal{E} \propto\left(C_{\omega}^{2}-\frac{\left(3-\alpha^{2}\right)}{128} f_{\rho}^{2}\right) n^{2}$ has the possibility of becoming negative for sufficiently large $f_{\rho}$. Furthermore the equation for the effective mass $M^{*}$ will ultimately be dominated by the $\rho$ vector-tensor and tensortensor contributions which will give rise to a negative value of $M^{*}$ (for positive $\left.f_{\rho} / g_{\rho}\right)$. Such problems arise in principle for the $\rho$, but only for densities $\gtrsim$ $15 n_{0}$ which are well beyond our range of interest and probably beyond the range of applicability of a simple baryon-meson picture. 
The Landau effective mass, $M_{L}^{*}$, is defined in terms of the velocity at the Fermi surface, $v_{F}$, and the single particle spectrum, $\epsilon(k)$, according to [39]

$$
M_{L}^{*}=\frac{k_{F}}{v_{F}}, \text { where } v_{F}=\left.\frac{\partial \epsilon(k)}{\partial k}\right|_{k=k_{F}} .
$$

The well known result $M_{L}^{*}=\left(k_{F}^{2}+M^{* 2}\right)^{\frac{1}{2}}$ is obtained in the MRHA. Here we shall study the modification of $M_{L}^{*}$ induced by the exchange diagram for the case of nuclear matter where the neutron and proton Fermi momenta are the same. In order to perform the calculation we need $\epsilon(k)$. This can conveniently be obtained by taking the functional derivative of the energy density with respect to the occupation probability, $\delta \mathcal{E} / \delta n(\mathbf{k})$, where, for nuclear matter, $n(\mathbf{k})=\left(4 \pi^{3}\right)^{-1} \theta\left(k_{F}-|\mathbf{k}|\right)$. The contributions of scalar and vector exchange to $\epsilon(k)$ have been given by Chin [3]. The contribution of the $\rho$ can be written as the sum of vector-vector, tensor-tensor and vector-tensor terms,

$$
\epsilon_{\rho}(k)=\epsilon_{\rho}^{\mathrm{vv}}(k)+\epsilon_{\rho}^{\mathrm{tt}}(k)+\epsilon_{\rho}^{\mathrm{vt}}(k)
$$

For nuclear matter these terms are

$$
\begin{aligned}
& \epsilon_{\rho}^{\mathrm{vv}}(k)=\left(\frac{g_{\rho}}{2}\right)^{2} \frac{3}{8 \pi^{2} E_{k}^{*}}\left[k_{F} E_{F}^{*}-M^{* 2} \ln \xi-\left(2+w_{\rho}\right) \frac{M^{* 3}}{k} J\left(w_{\rho}, \xi_{k}, \xi\right)\right], \\
& \epsilon_{\rho}^{\mathrm{tt}}(k)=-\left(\frac{f_{\rho}}{4 M}\right)^{2} \frac{1}{8 \pi^{2} E_{k}^{*}}\left[2 k_{F}^{3} E_{k}^{*}-3 M^{* 2}\left(5+\frac{1}{2} w_{\rho}\right)\left(k_{F} E_{F}^{*}-M^{* 2} \ln \xi\right)\right. \\
& \left.+\frac{12 M^{* 5}}{k} w_{\rho}\left(1+\frac{1}{8} w_{\rho}\right) J\left(w_{\rho}, \xi_{k}, \xi\right)\right], \\
& \epsilon_{\rho}^{\mathrm{vt}}(k)=\left(\frac{g_{\rho} f_{\rho}}{8 M}\right) \frac{9 M^{*}}{4 \pi^{2} E_{k}^{*}}\left[k_{F} E_{F}^{*}-M^{* 2} \ln \xi-\frac{M^{* 3}}{k} w_{\rho} J\left(w_{\rho}, \xi_{k}, \xi\right)\right],
\end{aligned}
$$

where $E_{k}^{* 2}=k^{2}+M^{* 2}, \xi_{k}=\left(k+E_{k}^{*}\right) / M^{*}$ and the other quantities are defined above. The derivative of $\epsilon$ is then easily evaluated to obtain the contribution to the Fermi velocity. 


\subsection{Results}

When the exchange diagram is included it is necessary to refit nuclear matter properties. In order to restrict the number of parameters, we take a $\sigma$ mass of $550 \mathrm{MeV}$ and fix the $\rho$-nucleon couplings. For the vector part we take $g_{\rho}^{2} / 4 \pi=$ $2.4\left(C_{\rho}^{2}=44.9\right)$ from $\pi N$ scattering [40], assuming $\rho$ universality. Reference [40] indicates a tensor-vector ratio, $f_{\rho} / g_{\rho}$, of 6.6 , which is at the upper end of currently accepted values [7]. We shall also consider the smaller ratio of 3.7 derived from the isovector anomalous magnetic moment of the nucleon in the vector dominance model; studies of nuclear systems in the Hartree-Fock approximation [12] favor the smaller value. The $\sigma$ and $\omega$ coupling constants which result from fitting the saturation properties are collected in table 6 . Since the net effect of exchange is repulsive, it is necessary to reduce the $\omega$ coupling and for $\mu / M=1.5, C_{\omega}^{2}$ is unreasonably small and, in fact, we were not able to obtain a satisfactory fit with the stronger tensor coupling. We also show in table 6 the compression moduli which show some modest changes from their previous values; for $\mu / M=0.79$ and $1.0, K$ is reduced, whereas in the other cases $K$ increases. In the last column we give the coefficient of the symmetry energy, $a_{\text {symm. }}$. Here the exchange contribution from the $\sigma$ $(\omega)$ is positive (negative), while the effect of the $\rho$ is quite small; the net contribution from exchange is large, $\sim+15 \mathrm{MeV}$, as is well known. Thus without the direct $\rho$ contribution $a_{\text {symm }} \sim 30 \mathrm{MeV}$ and, adding in the $\rho$, one obtains a value in the neighborhood of $40 \mathrm{MeV}$. Given that currently accepted values are in the range $27-35 \mathrm{MeV}$ [11, 42], our results are somewhat high particularly for $\mu / M<1$. 
The effect of exchange on the binding energy/particle as a function of density is shown in fig. 2. The dashed curves correspond to the weaker tensor $\rho$ coupling for which the effect of exchange is fairly small, although the impact of the increased symmetry energy is noticeable for neutron matter. The dash-dotted curves show the results for the stronger tensor coupling; the reduced values of $C_{\omega}^{2}$ here lead to a softening of the equation of state for densities beyond $0.43 \mathrm{fm}^{-3}$ in all cases. The parameters $C$ and $\widetilde{A}$ of eqs. (8) and (9) characterise the change in equilibrium density and compression modulus with the neutron excess parameter $\alpha$. The effect of exchange upon these quantities is case dependant, but typically the values of $C$ in table 2 are increased by $10 \%$ and the values of $\widetilde{A}$ are increased by $20 \%$.

In table 7 we show the individual contributions of the MRHA and exchange to the Fermi velocity (see eq. (22)). The exchange contribution arises from $\sigma$ (negative) and $\omega$ and $\rho$ (positive) contributions, none of which is insignificant. The total exchange contribution is about $5 \%$ (25\%) for the weak (strong) tensor coupling. In the weak case the net results for the Landau effective mass are quite close to those given in table 1 for the pure MRHA, whereas a reduction of $\sim 10 \%$ is obtained with the strong tensor coupling. In neither case are the exchange contributions to the effective mass sufficiently small that they can safely be ignored.

Turning to neutron stars, we find that properties given in table 3 are little changed when exchange effects are included using the weaker tensor $\rho$ coupling. With the stronger tensor coupling the equation of state is softer which leads to a modest increase in the central densities and a small decrease in the maximum masses. The predictions for $\mu / M=0.79$ and 1.0 become very 
similar, in particular the maximum mass is close to $2.26 M_{\odot}$. The predictions for $\mu / M=0.73$ and 1.25 are also similar and the maximum mass of $1.79 M_{\odot}$ is probably a little low in view of the expected effect of hyperons [18, 19]. The cooling of neutron stars by the direct Urca process is allowed in all cases, as was the case without exchange.

Summarizing this section, we have examined the matter contribution arising from the lowest order exchange diagram using the minimum number of mesons and found quite modest effects for neutron-rich systems, even with the strong tensor-vector ratio for the $\rho$ coupling. It is unlikely that inclusion of, for example, the pion field would qualitatively change this conclusion. Indeed rather small effects were obtained from the Fock terms in (pure matter) Hartree-Fock calculations [43] which included the pion as well as a number of baryon fields.

\section{Conclusions}

The main thrust of our work has been to examine the implications of different choices of the renormalization scale in neutron-rich matter and finite nuclei, following our previous work [2, 4] with nuclear matter. Physical results would be independant of this scale if we had embarked on a complete renormalization program which accounted for all the important physical effects, but, of course, we cannot claim that this is the case. Within the confines of the relativistic Hartree approximation we have previously found [4] that nuclear matter favors values of $\mu / M=0.79$ and 1.25 . The observed masses of neutron stars do not distinguish different values of $\mu / M$; however, for finite nuclei 
we find that the phenomenology with $\mu / M=0.79$ is favored since it provides rather reasonable agreement with the wide range of data considered. We can compare with the mean field approach (ref. [1] and references therein) which has the disadvantage that it excludes the vacuum which is an inherent feature of relativistic field theories. Provided $\sigma^{3}$ and $\sigma^{4}$ terms are included in the Lagrangian, this approach gives somewhat better agreement for finite nuclei, particularly as regards the single particle energies. However it must be borne in mind that non-relativistic calculations [44] show that correlations play an important role in determining the spectral strength distributions in actual nuclei.

Changing the renormalization scale $\mu / M$ from 1.0 to 0.79 can be viewed as choosing the effective three and four body scalar self-couplings to be zero at a point which correspond to $60 \%$ of equilibrium nuclear matter density, rather than in the vacuum. For $\mu / M<1$, the coefficient of the $\sigma^{4}$ term is negative and one can worry that the effective potential of the scalar field,

$$
U_{\mathrm{eff}}(\sigma)=\frac{1}{2} m_{\sigma}^{2} \sigma^{2}+\Delta \mathcal{E}\left(M-g_{\sigma} \sigma\right)
$$

has quite a shallow minimum [4] at the vacuum value of $\sigma=0$ for $\mu / M=0.79$. More troublesome is the fact that for $\sigma \rightarrow-\infty$ the effective potential is unbounded from below, $U_{\text {eff }} \rightarrow-\infty$, irrespective of the choice of the renormalization scale. This means that formally we are dealing with a local minimum rather than an absolute minimum in the energy. Thus, while the phenomenology is successful in explaining a large amount of data, unresolved theoretical issues remain.

We thank C.J. Horowitz for giving us a copy of his computer code for 
finite nuclei and for discussions pertaining thereto. We acknowledge partial support from the Department of Energy under contracts No. DE-FG0287ER40328, DE-AC02-83ER40105 and DE-FG02-88ER40388. A grant for computing time from the Minnesota Supercomputer Institute is gratefully acknowledged.

\section{References}

[1] B.D. Serot and J.D. Walecka, Advance in Nuclear Physics, Vol. 16, ed. J.W. Negele and E. Vogt (Plenum, NY, 1986); B.D. Serot, Quantum Hadrodynamics, Rep. Prog. Phys. 55 (1992) 1855.

[2] E.K. Heide and S. Rudaz, Phys. Lett. B262 (1991) 375.

[3] S.A. Chin, Ann. Phys. (NY) 108 (1977) 301.

[4] S. Rudaz, P.J. Ellis, E.K. Heide and M. Prakash, Phys. Lett. B285 (1992) 183.

[5] J.M. Lattimer, C.J. Pethick, M. Prakash and P. Haensel, Phys. Rev. Lett. 66 (1991) 2701; M. Prakash, M. Prakash, J.M. Lattimer and C.J. Pethick, Ap. J. Lett. 390 (1992) L77.

[6] M. Prakash, P.J. Ellis and J. Kapusta, Phys. Rev. C45 (1992) 2518;

R. Friedrich, K. Wehrberger and F. Beck, Phys. Rev. C46 (1992) 188.

[7] O. Dumbrajs et al., Nucl. Phys. B216 (1983) 277; J. Hamilton and G.C. Oades, Nucl. Phys. A424 (1984) 447. 
[8] J.M. Pearson, Phys. Lett. B271 (1991) 12.

[9] S. Shlomo and D.H. Youngblood, Phys. Rev. C47 (1993) 529.

[10] M.M. Sharma, The Compressibility of Nuclear Matter Revisited: the Third Derivative of EOS from Breathing Mode, Daresbury preprint DL/NUC/P323T, December 1991, unpublished.

[11] M. Prakash and K.S. Bedell, Phys. Rev. C32 (1985) 1118; M. Prakash and T.L. Ainsworth, Phys. Rev. C36 (1987) 346; I. Bombaci, T.T.S. Kuo and U. Lombardo, Phys. Rep., to be published.

[12] A. Bouyssy, J.-F. Mathiot, N. Van Giai and S. Marcos, Phys. Rev. C36 (1987) 380 .

[13] R.C. Tolman, Proc. Nat. Acad. Sci. USA 20 (1934) 3; J.R. Oppenheimer and G.M. Volkov, Phys. Rev. 55 (1939) 374.

[14] J.W. Negele and D. Vautherin, Nucl. Phys. A207 (1974) 298.

[15] G. Baym, C.J. Pethick and P. Sutherland, Ap. J. 170 (1971) 299.

[16] S. Rappaport and P.C. Joss, Ann. Rev. Astron. Astrophys. 22 (1984) 537.

[17] J.M. Weisberg and J.H. Taylor, Phys. Rev. Lett. 52 (1984) 1348; S.E. Thorsett, Z. Arzoumanian, M.M. McKinnon and J.H. Taylor, Ap. J. 405 (1993) L29.

[18] N.K. Glendenning, Ap. J. 293 (1985) 470; N.K. Glendenning, Phys. Rev. Lett. 57 (1986) 1120. 
[19] J.I. Kapusta and K.A. Olive, Phys. Rev. Lett. 64 (1990) 13; Nucl. Phys. B348 (1991) 345.

[20] M. Prakash, T.L. Ainsworth and J.M. Lattimer, Phys. Rev. Lett. 61 (1988) 2518.

[21] P. Haensel and J.L. Zdunik, Nature 340 (1989) 617.

[22] J.L. Friedman, J.R. Ipser and L. Parker, Ap. J. 304 (1986) 115; Phys. Rev. Lett. 62 (1989) 3015.

[23] J.M. Lattimer, M. Prakash, D. Masak and A. Yahil, Ap. J. 355 (1990) 241.

[24] C.J. Horowitz and B.D. Serot, Nucl. Phys. A368 (1981) 503; C.J. Horowitz and B.D. Serot, Phys. Lett. B140 (1984) 181.

[25] R.J. Perry, Phys. Lett. B182 (1986) 269.

[26] D.A. Wasson, Phys. Lett. B210 (1988) 41.

[27] D.A. Wasson, Nucl. Phys. A535 (1991) 456; D.A. Wasson and S.E. Koonin, Phys. Rev. D43 (1991) 3400.

[28] P.G. Blunden, Phys. Rev. C41 (1990) 1851.

[29] R.J. Furnstahl and C.E. Price, Phys. Rev. C41 (1990) 1792.

[30] P.G. Blunden, private communication.

[31] J.W. Negele, Phys. Rev. C1 (1970) 1260. 
[32] L.J. Tassie and F.C. Barker, Phys. Rev. 111 (1958) 940.

[33] W.R. Fox, Nucl. Phys. A495 (1989) 463.

[34] M. Rufa, P.-G. Reinhard, J.A. Maruhn, W. Greiner and M.R. Strayer, Phys. Rev. C38 (1988) 390.

[35] A. Bouyssy, S. Marcos and P. van Thieu, Nucl. Phys. A422 (1984) 541.

[36] Y.K. Gambhir, P. Ring and A. Thimet, Ann. Phys. 198 (1990) 132.

[37] I. Sick and J.S. McCarthy, Nucl. Phys. A150 (1970) 631.

[38] H. de Vries, C.W. de Jager and C. de Vries, At. Data and Nucl. Data Tables, 36 (1987) 495.

[39] G. Baym and S.A. Chin, Nucl. Phys. A262 (1976) 527.

[40] G. Höhler and E. Pietarinen, Nucl. Phys. B95 (1975) 210.

[41] P. Möller, W.D. Myers, W.J. Swiatecki, and J. Triener, At. Data and Nucl. Data Tables, 39 (1988) 225.

[42] J.M. Pearson, Y. Aboussir, A.K. Dutta, R.C. Nayak, M. Farine and F. Tondeur, Nucl. Phys. A528 (1991) 1.

[43] F. Weber and M.K. Weigel, Nucl. Phys. A493 (1989) 549; ibid. A505 (1989) 779.

[44] G.A. Rijsdijk, K. Allaart and W.H. Dickhoff, Nucl. Phys. A550 (1992) 159. 
Table 1

MRHA coupling constants and saturation properties

\begin{tabular}{|cccccc|}
\hline$\frac{\mu}{M}$ & $\frac{M_{\mathrm{sat}}^{*}}{M}$ & $K$ & $C_{\omega}^{2}$ & $C_{\sigma}^{2}$ & $C_{\rho}^{2}$ \\
& & $(\mathrm{MeV})$ & & & \\
\hline 0.73 & 0.73 & 157 & 132.8 & 279.4 & 82.5 \\
0.79 & 0.66 & 354 & 180.6 & 317.5 & 73.5 \\
1.00 & 0.73 & 461 & 137.7 & 215.0 & 81.6 \\
1.25 & 0.82 & 264 & 78.6 & 178.6 & 90.8 \\
1.50 & 0.86 & 189 & 51.2 & 175.6 & 94.4 \\
\hline
\end{tabular}

Table 2

Parameters specifying the saturation density and isobaric compression modulus of neutron-rich matter

\begin{tabular}{|ccccc|}
\hline$\frac{\mu}{M}$ & $C$ & $A$ & $B$ & $\widetilde{A}$ \\
\hline 0.73 & 1.62 & 3.36 & 5.45 & 2.09 \\
0.79 & 0.76 & 1.56 & 4.45 & 2.89 \\
1.00 & 0.54 & 1.05 & 2.16 & 1.10 \\
1.25 & 0.91 & 1.75 & 2.96 & 1.21 \\
1.50 & 1.25 & 2.39 & 3.64 & 1.25 \\
\hline
\end{tabular}


Table 3

Neutron star properties in the MRHA

\begin{tabular}{|ccccccc|}
\hline$\frac{\mu}{M}$ & $K$ & $\frac{M_{\max }}{M_{\odot}}$ & $R$ & $\frac{n_{c}}{n_{0}}$ & $\frac{n_{c}}{n_{0}}$ & $\Omega_{K}$ \\
& $(\mathrm{MeV})$ & & $(\mathrm{km})$ & $\left(M_{\max }\right)$ & $\left(1.44 M_{\odot}\right)$ & $\left(10^{4} \mathrm{~s}^{-1}\right)$ \\
\hline 0.73 & 157 & 2.18 & 11.2 & 6.2 & 2.6 & 0.96 \\
0.79 & 354 & 2.53 & 12.7 & 4.8 & 1.9 & 0.86 \\
1.00 & 461 & 2.30 & 12.1 & 5.3 & 2.0 & 0.88 \\
1.25 & 264 & 1.86 & 10.7 & 7.3 & 3.1 & 0.95 \\
1.50 & 189 & 1.59 & 9.9 & 9.1 & 4.5 & 0.99 \\
\hline
\end{tabular}

Table 4

Bulk properties of nuclei for $m_{\sigma}=550 \mathrm{MeV}$

\begin{tabular}{|c|rc|cc|ccc|}
\hline & \multicolumn{2}{|c|}{$\mathrm{O}$} & \multicolumn{2}{c|}{$\mathrm{Ca}$} & \multicolumn{3}{c|}{$\mathrm{Pb}$} \\
$\frac{\mu}{M}$ & $\frac{B E}{A}$ & $r_{c h}$ & $\frac{B E}{A}$ & $r_{c h}$ & $\frac{B E}{A}$ & $r_{c h}$ & $2 f \nu$ split \\
& $(\mathrm{MeV})$ & $f \mathrm{~m}$ & $(\mathrm{MeV})$ & $\mathrm{fm}$ & $(\mathrm{MeV})$ & $\mathrm{fm}$ & $(\mathrm{MeV})$ \\
\hline 0.73 & 9.42 & 2.66 & 9.52 & 3.42 & 8.33 & 5.57 & 1.09 \\
0.79 & 7.52 & 2.80 & 7.99 & 3.54 & 7.47 & 5.54 & 1.64 \\
1.00 & 6.08 & 2.64 & 6.92 & 3.38 & 6.70 & 5.40 & 1.31 \\
1.25 & 9.16 & 2.51 & 9.26 & 3.28 & 7.99 & 5.40 & 0.73 \\
1.50 & 10.59 & 2.46 & 10.31 & 3.24 & 8.56 & 5.43 & 0.50 \\
\hline Expt. & 7.98 & 2.73 & 8.45 & 3.48 & 7.86 & 5.50 & 1.77 \\
\hline
\end{tabular}


Table 5

Bulk properties of nuclei with $m_{\sigma}$ fitted

\begin{tabular}{|cc|cc|cc|cc|}
\hline & & \multicolumn{2}{|c|}{$\mathrm{O}$} & \multicolumn{2}{c|}{$\mathrm{Ca}$} & \multicolumn{2}{c|}{$\mathrm{Pb}$} \\
$\frac{\mu}{M}$ & $\begin{array}{c}m_{\sigma} \\
(\mathrm{MeV})\end{array}$ & $\begin{array}{c}\frac{B E}{A} \\
(\mathrm{MeV})\end{array}$ & $\begin{array}{c}r_{c h} \\
\mathrm{fm}\end{array}$ & $\frac{B E}{A}$ & $r_{c h}$ & $\frac{B E}{A}$ & $r_{c h}$ \\
$(\mathrm{MeV})$ & $\mathrm{fm}$ & $(\mathrm{MeV})$ & $f \mathrm{fm}$ \\
\hline 0.79 & 600 & 8.13 & 2.74 & 8.47 & 3.49 & 7.73 & 5.51 \\
1.00 & 450 & 4.03 & 2.79 & 5.35 & 3.49 & 5.82 & 5.45 \\
1.25 & 350 & 5.53 & 2.77 & 6.65 & 3.48 & 6.63 & 5.50 \\
\hline \multicolumn{2}{|c|}{ Experiment } & 7.98 & 2.73 & 8.45 & 3.48 & 7.86 & 5.50 \\
\hline
\end{tabular}

Table 6

MRHA + exchange coupling constants and saturation properties

\begin{tabular}{|ccrccc|}
\hline$\frac{\mu}{M}$ & $K$ & $C_{\omega}^{2}$ & $C_{\sigma}^{2}$ & $\frac{f_{\rho}}{g_{\rho}}$ & $\begin{array}{c}a_{\text {symm }} \\
(\mathrm{MeV})\end{array}$ \\
\hline \multirow{2}{*}{0.73} & 191 & 88.3 & 257.1 & 3.7 & 41.7 \\
& 204 & 57.9 & 212.0 & 6.6 & 38.6 \\
0.79 & 332 & 128.9 & 283.6 & 3.7 & 42.2 \\
& 274 & 96.4 & 247.1 & 6.6 & 39.7 \\
1.00 & 460 & 106.7 & 201.5 & 3.7 & 34.8 \\
& 421 & 80.9 & 186.6 & 6.6 & 34.4 \\
1.25 & 275 & 49.5 & 169.6 & 3.7 & 35.4 \\
& 280 & 26.3 & 149.5 & 6.6 & 34.6 \\
1.50 & 198 & 18.7 & 169.3 & 3.7 & 37.9 \\
\hline
\end{tabular}


Table 7

Landau effective masses in the MRHA+exchange approximation

\begin{tabular}{|c|ccc|ccc|}
\hline$\mu / M$ & \multicolumn{3}{|c|}{$f_{\rho} / g_{\rho}=3.7$} & \multicolumn{3}{c|}{$f_{\rho} / g_{\rho}=6.6$} \\
& $\begin{array}{c}\text { Contribution to } v_{F} \\
\text { MRHA }\end{array}$ & $M_{L}^{*} / M$ & exchange & & Contribution to $v_{F}$ & $M_{L}^{*} / M$ \\
& MRHA & exchange & \\
\hline 0.73 & 0.350 & 0.011 & 0.78 & 0.339 & 0.086 & 0.66 \\
0.79 & 0.378 & 0.021 & 0.70 & 0.364 & 0.089 & 0.62 \\
1.00 & 0.354 & 0.038 & 0.72 & 0.348 & 0.103 & 0.62 \\
1.25 & 0.322 & 0.023 & 0.81 & 0.318 & 0.095 & 0.68 \\
1.50 & 0.308 & 0.012 & 0.87 &. &. &. \\
\hline
\end{tabular}




\section{Figure Captions}

Fig. 1. The ratio of the third and second derivatives of the nuclear matter equation of state at the equilibrium point, $S / K$, versus the compression modulus, $K$. The shaded band is obtained from the breathing mode data [8] and the filled square is the value suggested by Sharma [10]. The MRHA points are labelled by the value of the renormalization scale, $\mu / M$.

Fig. 2. The binding energy/particle as a function of density for neutron and nuclear matter with four values of the renormalization scale. The full curves correspond to the MRHA and the dashed (dash-dotted) curves correspond to MRHA + exchange with the weaker (stronger) tensor $\rho$ coupling. The upper (lower) set of curves are for neutron (nuclear) matter.

Fig. 3. Occupied and unoccupied neutron levels near the Fermi energy in ${ }^{208} \mathrm{~Pb}$. The experimental data are compared with predictions for $\mu / M=0.79$, $1.0,1.25$.

Fig. 4. As for fig. 5, but for protons.

Fig. 5. Comparison of the experimental charge density [37 for ${ }^{16} \mathrm{O}$ with theoretical predictions for $\mu / M=0.79,1.0,1.25$.

Fig. 6 As for fig. 5, but for ${ }^{40} \mathrm{Ca}$. The data are from ref. 38.

Fig. 7 As for fig. 6, but for ${ }^{208} \mathrm{~Pb}$.

Fig. 8. The exchange or Fock diagram. In our calculations the dashed line represents the exchange of a $\sigma$-, $\omega$ - or $\rho$-meson. 